\section{P2-66 PHYSICAL ACTIVITY AND ITS ASSOCIATIONS WITH PREVALENCE OF OVERWEIGHT, HYPERTENSION, DIABETES AND ISCHAEMIC HEART DISEASE IN THE KADOORIE BIOBANK STUDY OF 0.5 MILLION PEOPLE IN CHINA}

doi:10.1136/jech.2011.142976i.1

${ }^{1} \mathrm{H}$ Du, D Bennett, ${ }^{* 2,3} \mathrm{~L} \mathrm{Li},{ }^{1} \mathrm{G}$ Whitlock, ${ }^{1} \mathrm{R}$ Collins, ${ }^{2} \mathrm{Y}$ Guo, ${ }^{1} \mathrm{R}$ Peto, ${ }^{2} \mathrm{~J}$ Chen, ${ }^{1} \mathrm{Z}$ Chen. ${ }^{1}$ University of Oxford, Oxford, UK; ${ }^{2}$ Chinese Centre for Disease Control, Beijing, China; ${ }^{3}$ Chinese Academy of Medical Sciences, Beijing, China

Introduction Physical activity is associated with cardiovascular risk in Western populations but evidence from the Chinese population is limited.

Methods We examined baseline data on over 500000 people aged 30-79 years who, during 2004-2008, were recruited into a prospective study from 10 regions in China. Information on physical activity was collected using an interviewer-administered questionnaire that enquired about the frequency, duration and intensity of work-related and leisure activities. Total physical activity (METhours/day) was calculated from the time reportedly spent on each activity and the published estimate of energy expenditure per unit time (MET) for that specific activity. Body mass index, blood pressure and capillary glucose were measured. Outcomes of interest included overweight, hypertension, diabetes status (which were all defined using established criteria) and self-reported history of ischaemic heart disease (IHD). Logistic regression analyses were performed to assess the association of physical activity with each outcome.

Results Of the 456118 participants who were free of major chronic diseases other than IHD, the mean age was 51 years and $60 \%$ were women. Physical activity was significantly inversely associated with overweight, hypertension, diabetes and IHD, after adjustment for age, sex, region, education, income and smoking status. Comparing the bottom $20 \%$ vs the top $20 \%$ of physical activity, the adjusted ORs were: 1.25 (95\% CI 1.23 to 1.28$)$ for overweight, 1.23 (1.19 to 1.26) for hypertension, 1.72 (1.63 to 1.82$)$ for diabetes and 1.59 (1.46 to 1.73) for IHD (each $\mathrm{p}<0.0001$ ).

Conclusion In Chinese adults, low physical activity is associated with an increased prevalence of overweight, hypertension, diabetes and IHD.

\section{P2-67 REVERSING THE EPIDEMIC OF CIRRHOSIS MORTALITY: EVIDENCE FROM BIRTH COHORT ANALYSIS}

doi:10.1136/jech.2011.142976i.2

K Dunbar, ${ }^{*}$ I Crombie. University of Dundee, Tayside, UK

Introduction Alcohol is one of the top 10 risk factors for death in middle and high income countries. As average incomes in developing nations rise, disposable income will become available for alcohol. This could result in epidemics of alcohol related illness. Average national alcohol consumption has been shown to correlate with national rates of liver cirrhosis mortality in developed nations. Many countries have reversed trends of increasing cirrhosis mortality over short time periods. An improved understanding of the mechanism of these reversals would be of great benefit to policy makers.

Methods Mortality data for developed nations over the past 50 years were investigated by the birth cohort approach. Data were obtained from WHO. Age specific mortality rates were plotted against age at death for each birth cohort. This permitted a comparison between age specific rates throughout the life of each birth cohorts.

Results In countries where an increasing trend in alcohol consumption was sharply reversed, liver cirrhosis death rates fell dramatically. Further it did so simultaneously in all adult age groups. Subsequently each birth cohort continued to experience almost uninterrupted falls in age specific death rates. Moreover those birth cohorts which were experiencing high rates of mortality fell faster than those experiencing low rates. This may indicate that birth cohorts with high rates had a higher attributable fraction due to alcohol consumption.

Conclusion This study has shown that dramatic and sustained falls in cirrhosis mortality are possible. The challenge for governments is to implement policies to achieve this.

\section{P2-68 TRENDS IN CHRONIC DISEASE MORTALITY IN BRAZIL, 1996-2007}

doi:10.1136/jech.2011.142976i.3

${ }^{1}$ B Duncan, ${ }^{*}{ }^{1} \mathrm{M}$ I Schmidt, ${ }^{2} \mathrm{G}$ Mendonça, ${ }^{1} \mathrm{~A}$ Stevens. ${ }^{1}$ Universidade Federal do Rio Grande do Sul, Porto Alegre, Rio Grande do Sul, Brazil;, ${ }^{2}$ Universidade Estadual do Rio de Janeiro, Rio de Janeiro, Rio de Janeiro, Brazil

Background In recognition of the large burden and economic impact of non-communicable chronic diseases (NCDs), especially in low and middle income countries, the WHO has proposed a goal of an additional $2 \%$ reduction in mortality rates per year over current trends, and the United Nations is holding a High level Meeting on NCDs in September, 2011.

Objective To describe recent NCD mortality trends in Brazil, a middle-income country, taking into account recent improvements in death reporting.

Methods We obtained ICD-10 coded mortality data from the Brazilian mortality information system and population denominators from the Brazilian Institute of Geography and Statistics (IBGE). IBGE enumerated the population in 1996 and 2000, and extrapolated estimates for 2007. We redistributed ill-defined causes of death equally across all non-external cause deaths. We corrected for underreporting of deaths based on the ratio of expected to observed deaths. Expected deaths were estimated by IBGE, on the basis of life table analyses. Mortality rates were age-adjusted to the world population standard.

Results NCDs now account for $72 \%$ of deaths. In unadjusted analyses, corrected NCD mortality rates increased 5\% over the 12 year period. Age-adjusted rates declined 1.8\%/yr. Declines were greatest for chronic respiratory diseases $(2.8 \% / \mathrm{yr})$ and cardiovascular diseases (3.5\%/yr). Though declines occurred in all regions, 2007 rates are greatest in Brazil's poorest regions, where diabetes deaths have increased markedly.

Conclusion The decline in NCD mortality observed in Brazil demonstrates that a $2 \%$ decline/year is feasible, and encourages further public health action.

\section{P2-69 METABOLIC SYNDROME AND RISK OF BRAIN TUMOUR IN A LARGE POPULATION-BASED COHORT STUDY}

doi:10.1136/jech.2011.142976i.4

${ }^{1} \mathrm{M}$ Edlinger, ${ }^{*}{ }^{2,3} \mathrm{~T}$ Bjørge, ${ }^{4} \mathrm{~J}$ Manjer, ${ }^{5} \mathrm{P}$ Stattin, ${ }^{1} \mathrm{H}$ Ulmer. ${ }^{1}$ Medical University Innsbruck, Innsbruck, Austria; ${ }^{2}$ University of Bergen, Bergen, Norway; ${ }^{3}$ Norwegian Institute of Public Health, Oslo, Norway; ${ }^{4}$ Skåne University Hospital Malmö and Lund University, Malmö, Sweden; ${ }^{5}$ Umeå University, Umeå, Sweden

Background There are few established determinants of brain tumour. We assessed among adults the risk of brain tumour in relation to metabolic syndrome factors.

Methods 580000 subjects from Sweden, Austria, and Norway were followed for a median of 10 years (Me-Can). Brain tumour information was obtained from national cancer registries. The factors of metabolic syndrome, body mass index, blood pressure, and blood levels of glucose, cholesterol, and triglycerides, were analysed in 